\title{
IMPACTO DO INTERNAMENTO NA REDE NACIONAL DE CUIDADOS CONTINUADOS INTEGRADOS NA MELHORIA DOS NÍVEIS DA CAPACIDADE FUNCIONAL DOS UTENTES
}

\section{IMPACT OF INTERNATION ON THE NATIONAL NETWORK OF CONTINUOUS INTEGRATED CARE IN IMPROVING USER FUNCTIONAL CAPACITY LEVELS}

\author{
Rosa Martins ${ }^{1}$ \\ Tânia Henriques ${ }^{2}$ \\ Nélia Carvalho ${ }^{3}$
}

\begin{abstract}
Resumo: A Rede Nacional de Cuidados Continuados Integrados (RNCCI) tem por missão prestar os cuidados adequados, de saúde e apoio social, a todas as pessoas que, independentemente da idade, se encontrem em situação de dependência. Desta forma, torna-se emergente avaliar o impacto do internamento na RNCCI, na melhoria dos níveis de dependência dos utentes internados e analisar a sua relação com as variáveis sócio demográficas, clínicas e de contexto familiar.
\end{abstract}

Métodos: Desenhou-se uma investigação de perfil quantitativo, descritivo e exploratório, com dois momentos de avaliação, admissão e alta clínica, permitindo avaliar a evolução da condição de saúde (incapacidade funcional) dos participantes da amostra. Esta é do tipo não probabilística por conveniência, constituída por 160 pessoas internadas em duas unidades da RNCCI do distrito de Viseu. Para a mensuração das variáveis utilizou-se um instrumento de colheita de dados que integra uma secção de caracterização sócio demográfica, clínica, de contexto familiar e o Índice de Barthel.

Resultados: O impacto do internamento foi positivo na melhoria dos níveis de incapacidade funcional, uma vez que os valores encontrados na admissão versus alta foram respetivamente: sem incapacidade (0\% vs 10\%), incapacidade ligeira, (2,5\% vs 7,5\%), incapacidade moderada (20\% vs 30\%) e incapacidade grave $(77,5 \%$ vs $52,5 \%$ ). Verificámos ainda que, os utentes com maior capacidade funcional eram os detentores do $2^{\circ}$ ciclo de escolaridade $(p=0,005)$, os que auferiam rendimentos mensais superiores a $500 €(p=0,023)$, os que realizaram programas de reabilitação com 10 e mais horas semanais $(p=0,004)$ e que apresentavam integridade cutânea $(p=0,029)$.

\footnotetext{
${ }^{1}$ Docente da Escola Superior de Saúde do IP de Viseu- rmartins.viseu@gmail.com

${ }^{2}$ Enfermeira de uma UCC do Distrito de Viseu

${ }^{3}$ Estudante da Escola Superior de Saúde do IP de Viseu
} 
Conclusão: $O$ internamento na RNCCI tem impacto positivo na funcionalidade dos Utentes, dada a melhoria significativa dos níveis de dependência no momento da alta, comparativamente com o momento da admissão.

Palavras-chave: Impacto do internamento; RNCCI, (in)capacidade funcional Enfermeiro Especialista de Reabilitação; admissão; alta.

\begin{abstract}
The National Integrated Continuing Care Network $(N I C C N)$ is responsible for providing adequate health care and social support to all persons, regardless of age, in a situation of dependency. Thus, it is emerging to evaluate the impact of hospitalization on NICCN in improving levels of dependence of hospitalized patients and to analyze their relationship with sociodemographic, clinical and family context variables.

Methods: A qualitative, descriptive and exploratory profile investigation was designed, with two moments of evaluation, admission and clinical discharge, allowing the evaluation of the health condition evolution (functional disability) of the sample participants. This is a non-probabilistic convenience smapling, consisting of 160 people hospitalized in two units of the NICCN of the district of Viseu. For the measurement of the variables, a data collection instrument was used that integrates a socio-demographic, clinical, family context and Barthel Index.

Results: The impact of hospitalization was positive in improving functional disability levels, since the values found in admission versus discharge were: no disability (0\% vs $10 \%)$, mild disability $(2.5 \%$ vs $7.5 \%)$, moderate disability (20\% vs $30 \%)$ and severe disability (77.5\% vs $52.5 \%)$. We also verified that the users with the highest functional capacity were the holders of the 2 nd cycle of schooling ( $p$ $=0.005)$, those who received monthly incomes higher than $500 €(p=$ $0.023)$, those who performed rehabilitation programs with more than 10 hours weekly $(p=0.004)$ and had cutaneous integrity $(p=0.029)$.

Conclusion: The hospitalization in the NICCN has a positive impact on the functionality of the users, given the significant improvement in the levels of dependence at discharge, compared to the time of admission.
\end{abstract}

Keywords: : Impact of hospitalization; NICCN; functional (in)capacity; Nurse Rehabilitation Specialist; admission; Hospital discharge. 


\section{INTRODUÇÃO}

O cenário demográfico atual em Portugal remete-nos para a necessidade de mudanças no paradigma da organização dos cuidados de saúde. Deparamo-nos com uma população cada vez mais envelhecida, dependente e com necessidade de um maior acompanhamento ao longo do seu ciclo de vida, tal como é referenciado pelo Instituto Nacional de Estatística (INE) em 2016.

Sabemos pelos estudos que têm sido desenvolvidos sobre esta temática, que é em fases mais avançadas da vida que este acompanhamento se verifica imprescindível, uma vez que se associa ao declínio da capacidade funcional. Lopes, (2014), diz-nos que é nos grupos etários mais elevados que se encontra um maior número de indivíduos em situação de dependência, pelo que se torna fundamental avaliar esses níveis com o objetivo major de definir um modelo de intervenção adequado.

Assim, e numa tentativa de resposta às questões referidas, é criada a Rede Nacional de Cuidados Continuados Integrados (RNCCI) pelo Decreto-Lei n. ${ }^{\circ}$ 101/2006, de 6 de Junho, que se constitui como um modelo organizativo e funcional (...) "que tem por base promover a continuidade da prestação de cuidados de saúde e apoio social com responsabilidades de intervenção no melhor interesse do cidadão: o Serviço Nacional de Saúde e o Sistema de Segurança Social" (Relatório da RNCCI, 2015:4). O seu objetivo geral centra-se na prestação de cuidados continuados integrados a pessoas que, independentemente da idade, se encontrem em situação de dependência a qual se concretiza através dos seguintes objetivos:

Melhoria das condições de vida e bem-estar das pessoas em situação de dependência, através da prestação de cuidados continuados de saúde e de apoio social.

$\checkmark$ A manutenção das pessoas com perda de funcionalidade ou em risco de perder no domicílio, sempre que possam ser garantidos os cuidados terapêuticos e o apoio social necessários à provisão e manutenção de conforto e qualidade de vida;

$\checkmark \mathrm{O}$ apoio, o acompanhamento e o internamento tecnicamente adequados à respetiva situação;

$\checkmark$ A melhoria contínua da qualidade na prestação de cuidados continuados de saúde e de apoio social;

$\checkmark \mathrm{O}$ apoio aos familiares ou prestadores informais, na respetiva qualificação e na prestação dos cuidados; 
$\checkmark$ A articulação e coordenação em rede dos cuidados em diferentes serviços, setores e níveis de diferenciação;

$\checkmark$ A prevenção de lacunas em serviços e equipamentos, pela progressiva cobertura nacional, das necessidades das pessoas em situação de dependência em matéria de cuidados continuados integrados. (RNCCI - Manual do Prestador, 2011:8).

As projeções relativas ao Índice de Envelhecimento apontam para um aumento substancial até ao ano de 2060, podendo vir a atingir o valor de 307 idosos por cada 100 jovens, num cenário central, 464 idosos por cada 100 jovens num cenário baixo ou aumentar para 287 idosos por cada 100 jovens num cenário alto (INE, 2016).

As situações de dependência não são exclusivas de um determinado grupo etário, uma vez que existem pessoas dependentes de todas as idades, contudo a maior prevalência observa-se claramente na população mais idosa. Enquanto nas faixas etárias mais jovens, a dependência aparece na maioria dos casos associada a situações de deficiência congénita ou adquirida, no caso dos idosos, a dependência, é muitas vezes, uma consequência do processo gradual de envelhecimento humano. Na medida em que a idade cronológica aumenta o indivíduo, naturalmente, tende a tornar-se menos ativo, sendo que as suas capacidades físicas são reduzidas e, com isso, observamos evidências do aparecimento de alterações físicas psicológicas e sociais. As doenças crónicas adquirem neste contexto maior relevo e a redução do nível de atividade física aceleram estes processos, fazendo com que os idosos constituam o grupo mais suscetível à incapacidade funcional, constituindo-se esta um importante preditor de mortalidade.

A Dependência, tal como é conceituada pela legislação portuguesa, (D.L. n. ${ }^{\circ}$ 265/99 de 14 de Julho e o D.L. n. ${ }^{\circ} 309-A / 2000$ de 30 de Novembro), é a condição em que as pessoas “(...) não possam praticar com autonomia os atos indispensáveis à satisfação das necessidades básicas da vida quotidiana, carecendo da assistência de outrem." O Quarto Inquérito Nacional de Saúde, de 2015/2016, diz que a população residente que declarou estar sempre acamada, ou limitada à sua casa para se movimentar (...) tem uma expressão significativa representando uma acréscimo de cerca de $35 \%$, em relação ao último inquérito realizado. Estes dados permitem-nos afirmar que "o acréscimo do número de dependentes é justificado (...) em grande parte, pelo processo de envelhecimento que acarreta, naturalmente, um decréscimo de capacidades de várias ordens", ou seja, a dependência surge quando uma pessoa apresenta uma perda mais ou menos acentuada da sua autonomia 
funcional e necessita da ajuda de outra pessoa (Saraiva, 2011). Trata-se de um processo incapacitante pelo qual uma determinada condição (aguda ou crónica) afeta a funcionalidade dos idosos e o desempenho das atividades de vida diárias.

O relatório da Organização Mundial de Saúde (2011) traz dados sobre a incapacidade no mundo, que estão relacionados à incidência, prevalência, gravidade, duração e mortalidade para mais de 130 condições de saúde em 17 sub-regiões do mundo, estimando que 15,3\% da população mundial, cerca de 978 milhões de pessoas de uma estimativa de 6,4 bilhões em 2004 apresentava moderada ou grave incapacidade, enquanto $2,9 \%$ ou cerca de 185 milhões de pessoas apresentavam incapacidade grave.

A dependência tem sido avaliada a nível funcional, com base na capacidade e autonomia de execução das atividades de vida diária básicas e atividades instrumentais, uma vez que conhecer o grau de incapacidade funcional dos indivíduos com dependência é fundamental, dado que ajuda a determinar os cuidados necessários.

Apesar das mudanças estruturais das famílias, evidenciadas nos últimos anos em Portugal, estas continuam a ter um papel importantíssimo, no apoio dos seus membros, continuando a ser considerada a principal fonte de suporte nos cuidados diretos, no apoio psicológico e nos contactos sociais à pessoa idosa dependente (Pinto \& Martins 2013). Estes dados são confirmados pelo Relatório de Monitorização da RNCCI, que mostram que previamente ao internamento, $82 \%$ da origem de apoio prestado era atribuída aos familiares. Há portanto uma necessidade premente de métodos inovadores e imaginativos, que possam contribuir para uma atenção ao idoso/pessoa dependente, em bases humanísticas e, ao mesmo tempo, compatíveis com a realidade socioeconómica do país. O objetivo final deve ser sempre a manutenção contribuindo, ativamente, para ela, e mantendo o grau de autonomia (e dignidade) dos indivíduos pelo maior tempo possível.

\section{MATERIAL E MÉTODOS}

Foi desenvolvido um desenho de investigação de perfil quantitativo, descritivo e exploratório, com dois momentos de avaliação, admissão e alta clínica, permitindo avaliar a evolução da condição de saúde (incapacidade funcional) dos participantes da amostra. Esta era constituída por 160 pessoas internadas em duas unidades da RNCCI do 
distrito de Viseu. As questões de investigação que emergiram neste estudo são: Qual a dependência funcional dos doentes internados em RNCCI? Em que medida o internamento nestas unidades tem impacto na sua reabilitação? Será que as variáveis sociodemográficas, familiares e clínicas estão relacionadas com a dependência funcional apresentada pelos utentes?"

Os critérios de seleção estabelecidos foram: estar institucionalizado; ter sido submetido a avaliação do nível de dependência através da utilização da escala de Barthel, no momento da admissão e alta e um internamento mínimo de 60 dias;

A colheita de dados decorreu entre Maio e Setembro de 2016 e o instrumento de colheita de dados continha um grupo de questões de caracterização sociodemográfica, um segundo de caracterização familiar, um terceiro de variáveis clínicas, e por fim uma Escala de Barthel já testada e validada para a população portuguesa por Sequeira (2007).

Todos os procedimentos foram efetuados segundo uma rigorosa conduta ética, (com autorizações por parte dos Provedores e Diretores das Instituições e respetivas Comissões de Ética), garantindo-se o anonimato e confidencialidade dos dados recolhidos. O tratamento estatístico foi efetuado através do programa Statiscal Package Social Science versão 20.0 para o Windows e Word Microsoft, e foi processado utilizando estatística descritiva e estatística inferencial.

\section{RESULTADOS}

A amostra utilizada neste estudo é constituída por 160 participantes, sendo 64 do sexo feminino e 96 do sexo masculino. A idade da amostra total oscila entre um valor mínimo de 53 e um máximo de 92 anos, correspondendo-lhe uma idade média de 74,83 anos, um desvio padrão de 10,24 e um coeficiente de variação de $13,68 \%$, o que nos indica a existência de uma baixa dispersão em torno da média. A média de idades para o sexo feminino $(\mathrm{M}=79,31)$ é ligeiramente superior à do sexo masculino $(M=71,83)$. São maioritariamente casados/união de facto $(60,0 \%)$, e viúvos $(20,0 \%)$, apresentam níveis de escolaridade genericamente baixos, uma vez que $67,5 \%$ dos inquiridos tem entre 4 a 6 anos de escolaridade, seguindo-se os que os que não têm qualquer habilitação $(27,5 \%)$ e nenhum elemento possui habilitação de nível superior. Quanto à situação profissional, verificamos que a maioria se encontra reformado $(87,5 \%)$ ou desempregado $(10,0 \%)$ e o rendimento mensal oscila entre 251 a $500 €$ para $50 \%$, entre $501-1000 €$ para $22,5 \%$ e 
acima de $1000 €$ apenas $5 \%$. Vimos ainda que 50\% dos indivíduos vivem com o cônjuge, $32,5 \%$ sozinhos e $20,0 \%$ com os filhos/outros familiares. De referir que nesta questão surgiram respostas múltiplas, ou seja, sujeitos que simultaneamente habitavam com cônjuges filhos e netos. A tendência distributiva verificada entre homens e mulheres apresenta algumas diferenças, uma vez que as mulheres vivem mais sozinhas $(43,8 \%)$ enquanto os homens vivem maioritariamente com o cônjuge $(58,3 \%)$, o que de algum modo está em consonância com os dados demográficos dos últimos censos. O principal responsável pelo internamento destes utentes foram os filhos $(67,5 \%)$ e cônjuge $(17,5 \%)$ e ainda os vizinhos e amigos para 10\% dos participantes. Quanto às visitas, 85,0\% dos utentes diz ser visitado pelos filhos e cônjuge porém há que registar que $15 \%$ dos inquiridos refere não ser visitado por ninguém. Encontram-se ainda bastante satisfeitos com a ajuda que recebem da família (70\%) e com o modo como estes demonstram o seu afeto $(40,0 \%)$. De uma forma mais moderada (apenas algumas vezes) mostram satisfação com a forma como a família discute assuntos de interesse comum $(40,0 \%)$ e soluciona problemas (65\%). Maior desagrado encontramos no item relativo ao tempo que dedicam à família $(62,5 \%)$. Sobre a funcionalidade familiar $42,5 \%$ dos utentes acha que a sua família é moderadamente funcional, $32,5 \%$ altamente funcional e $25,0 \%$ refere disfunções acentuadas. Observámos que os homens tem perceções mais positivas (37,5\%), enquanto as mulheres se inserem essencialmente em valores moderados $(56,2 \%)$.

O quadro clinico que caracteriza os nossos participantes revela que $84,6 \%$ não teve internamentos nos últimos 6 meses, contudo aqueles que os tiveram $(15,4 \%)$ tinham estado em unidades de convalescença e de média duração. Os diagnósticos principais foram a doença vascular cerebral $(42,9 \%)$, as fraturas $(28,6 \%)$ e "outras" $(22,5 \%)$ onde estão incluídas as doenças respiratórias e metabólicas. O diagnóstico secundário de 95,0\% dos utentes estava associado á HTA (57,9\%), diabetes mellitus tipo II $(36,8 \%)$, doença cardíaca $(28,9 \%)$ e doença vascular cerebral $(26,3 \%)$. A terapia de reabilitação é fundamental nos utentes com estas características e por isso não nos surpreendeu que 95,0\% dos inquiridos a tenham feito com uma média semanal que oscilou entre 6 e 12 horas. Constatamos ainda que 35\% da amostra total apresentava alterações da integridade cutânea, em percentagens similares entre homens $(37,5 \%)$ e mulheres $(31,2 \%)$, sendo estas principalmente, 
úlceras de pressão $(57,1 \%)$, úlceras venosas $(28,6 \%)$ e lesões traumáticas $(14,3 \%)$.

Relativamente à dor, constatamos que no momento da admissão $25 \%$ dos Utentes não refere dor, $60 \%$ apresenta dor leve e $15 \%$ dor moderada. Já no momento da alta verificam-se algumas melhorias uma vez que $42,5 \%$ dos Utentes não tem dor, $42,5 \%$ tem dor leve e $12,5 \%$ dor moderada, o que revela uma maior sensibilização por parte dos profissionais de saúde no seu controle.

Considerando a variável central do estudo ou seja, a incapacidade funcional dos utentes, pudemos constatar (cf. Gráfico 1) que no momento da sua admissão, nenhum deles era independente, 2,5\% apresentava incapacidade ligeira, $20 \%$ incapacidade moderada e $77,5 \%$ apresentava incapacidade grave, relevando estes dados os elevados níveis de dependência caracterizadores desta tipologia de unidades. As incapacidades funcionais estavam associadas sobretudo á higiene, utilização do WC, banho, mobilidade, subir e descer escadas e vestir.

No momento da alta ( \pm 90 dias após admissão) a realidade funcional dos participantes sofreu modificações, uma vez que $10 \%$ apresentava independência total, 7,5\% apresentava incapacidade ligeira e 30\% incapacidade moderada. Refira-se ainda assim, que 52,5\% mantinha incapacidade grave, contudo são notórias as melhorias, o que nos permite afirmar que o internamento na RNCCI tem impacto positivo na funcionalidade dos Utentes.

\section{Gráfico 1}

Evolução nos níveis de incapacidade funcional dos Participantes

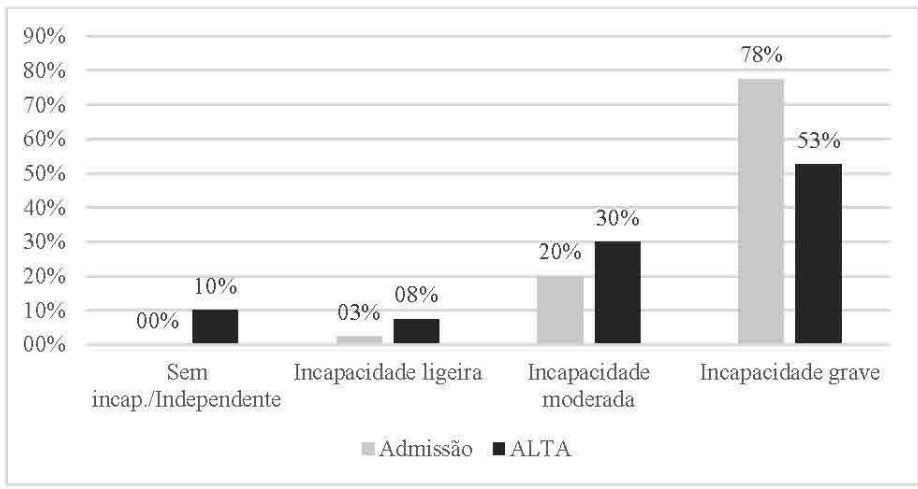

Explorando as relações entre as principais variáveis em estudo, verificamos associações com significância estatística apenas nas variáveis 
escolaridade, rendimentos mensais, horas dos programas de reabilitação e integridade cutânea, apresentando-se com maior capacidade funcional os participantes detentores do $2^{\circ}$ ciclo de escolaridade $(\mathrm{p}=0,005)$, os que auferiam rendimentos mensais superiores a $500 €(\mathrm{p}=0,023)$, os que realizaram programas de reabilitação com mais de 10 horas semanais $(\mathrm{p}=0,004)$ e que apresentavam integridade cutânea $(\mathrm{p}=0,029)$.

Já o género, idade, estado civil, situação profissional, funcionalidade familiar e tempo de internamento, mostraram não se correlacionar de forma significativa $(p>0,05)$ com a incapacidade funcional destes Utentes.

\section{DISCUSSÃO DOS RESULTADOS}

Considerando as características sociodemográficas da amostra, observamos que estão alinhadas com muitos outros estudos realizados em Portugal com população dependente (Filipe, 2015; Gonçalves, 2013; Petronilho et all. 2017).

Trata-se de uma amostra maioritariamente composta por idosos ( $M=74,83$ anos), confirmando a correlação expectável entre a idade mais avançada e o aumento do nível de dependência. São maioritariamente homens (60\%) casados (60\%), o que contraria a tendência frequentemente referida da «feminização» (Relatório de Monitorização da RNCCI; 2016). Apresentam baixos níveis de escolaridade (4 a 6 anos de escolaridade), maioritariamente $(87,5 \%)$ reformados e com um rendimento mensal que oscila entre 251 a $500 €$. Estes dados corroboram também os de Pinto e Martins (2013), ao afirmarem que os baixos níveis de escolaridade e rendimentos mensais podem dificultar a consciencialização para as necessidades de cuidado com a saúde ao longo da vida, a adesão ao tratamento e manutenção de estilo de vida saudável que limite a ação de indicadores de risco.

Vivem com o cônjuge (50\%), sozinhos (32,5\%) e com os filhos/outros familiares $(20, \%)$. A tendência distributiva verificada entre homens e mulheres apresenta algumas diferenças, uma vez que as mulheres vivem mais sozinhas $(43,8 \%)$ enquanto os homens vivem maioritariamente com o cônjuge (58,3\%), o que de algum modo está em consonância com os dados demográficos dos últimos censos (INE, 2016). Encontram-se bastante satisfeitos com a ajuda que recebem da família (70\%), mas entendem que esta lhe deveria dedicar mais tempo (62,5\%). Estes resultados não se podem dissociar e são consonantes com a perceção 
revelada sobre a funcionalidade familiar, uma vez que $42,5 \%$ dos participantes acha que a sua família é moderadamente funcional, 32,5\% altamente funcional e 25,0\% refere disfunções acentuadas. Observámos ainda que os homens tem perceções mais positivas que as mulheres. De facto sabemos que quando a família é confrontada com novos problemas ou exigências, tenta manter o equilíbrio utilizando as capacidades e os recursos que possui. Assim a família disfuncional tem mais dificuldade em manter o equilíbrio utilizando os seus recursos/estratégias de coping, enquanto uma família equilibrada/funcional se caracteriza por uma maior proximidade emocional, maior respeito pela autonomia e identidade, bem como pelo estabelecimento claro de fronteiras entre as gerações (Ferreira \& Pereira, 2016)

Clinicamente, $84,6 \%$ dos utentes não teve internamentos nos últimos 6 meses, contudo aqueles que os tiveram $(15,4 \%)$ tinham estado em unidades de convalescença e de média duração. Os diagnósticos principais foram a doença vascular cerebral $(42,9 \%)$, e as fraturas $(28,6 \%)$, e o diagnóstico secundário de $95,0 \%$ dos utentes estava associado á HTA $(57,9 \%)$, diabetes mellitus tipo II $(36,8 \%)$, doença cardíaca $(28,9 \%)$ e doença vascular cerebral $(26,3 \%)$. Os dados publicitados pela DGS (2013) referem que a prevalência do AVC aumenta com a idade, sendo uma das principais causas de grave deficiência e morte na União Europeia incluindo Portugal. Também Barbosa (2014) e Veiga (2015) afirmam que as doenças cardiovasculares, hipertensão, AVC, diabetes, entre outras, são as patologias que mais afetam a população idosa e as doenças crónicas constituem efetivamente um fator de risco para incapacidade funcional de idosos.

A terapia de reabilitação é fundamental nos utentes com estas características e por isso não nos surpreendeu que $95,0 \%$ dos inquiridos a tenham feito com uma média semanal que oscilou entre 6 e 12h.Constatamos que existe diferença estatisticamente significativa tanto no momento da admissão como da alta, demonstrando que são os utentes que fizeram 10 e mais horas de reabilitação semanal aqueles que apresentam maior capacidade funcional, como seria de esperar, dado que a Reabilitação é um fator preponderante na promoção da capacidade funcional ( Lourenço, 2011; Ferreira e Pereira, 2016).

Constatamos ainda que $35 \%$ da amostra total apresentava alterações da integridade cutânea, em percentagens similares entre homens (37,5\%) e mulheres $(31,2 \%)$, sendo estas principalmente úlceras de pressão $(57,1 \%)$, úlceras venosas $(28,6 \%)$ e lesões traumáticas $(14,3 \%)$. Este achado vem corroborar os resultados do estudo de Lino (2013) quando 
afirmam que "a presença de ferida crónica acarreta diversas limitações ao seu portador, desde a perda de mobilidade que leva à diminuição do nível de autonomia pessoal e da capacidade funcional, advindo a dependência de outrem.

Relativamente à dor, constatamos que no momento da admissão, $25 \%$ dos Utentes não refere dor, $60 \%$ apresenta dor leve e $15 \%$ dor moderada. Já no momento da alta verificam-se algumas melhorias uma vez que $42,5 \%$ dos Utentes não tem dor, $42,5 \%$ tem dor leve e $12,5 \%$ dor moderada, o que revela uma maior sensibilização por parte dos profissionais de saúde no seu controle. Relacionando este fator com a incapacidade funcional, observamos que não existe associação estatisticamente significativa. Estes dados estão em dissonância com os apresentados por Petronilho et all. (2017), que refere que a dor e respetiva intensidade como o maior preditor de incapacidade funcional.

Considerando os níveis de incapacidade funcional avaliados pudemos constatar que no momento da sua admissão, não existia nenhum elemento da amostra sem incapacidade ou seja nenhum era totalmente independente, apenas 2,5\% apresentava incapacidade ligeira, 20\% incapacidade moderada e 77,5\% apresentava incapacidade grave. Estes dados diferem do estudo apresentado por Filipe (2015) que conclui que no domínio motor, nas dimensões de autocuidado, controle de esfíncteres, transferências e locomoção os idosos, a maioria é independente; poucos apresentaram dependência mínima ou moderada e há uma minoria de dependência completa em algumas tarefas. No momento da alta a realidade funcional dos utentes era diferente, uma vez que já existiam $10 \%$ sem incapacidade (independentes), 7,5\% apresentava incapacidade ligeira, 30\% incapacidade moderada, e 52,5\% incapacidade grave. Estes resultados veem reforçar os de Veiga (2015), e Vieira et al. (2015) quando nos dizem que todos os participantes dos seus estudos, mostraram melhorias significativas no seu desempenho, desde a admissão até ao momento da alta, havendo uma diminuição dos graus de dependência e consequente aumento de independência e reabilitação funcional. Similarmente o Relatório de monitorização da RNCCI (2015) chama a atenção para o facto dos utentes integrarem grupos etários elevados o que pode condicionar o sucesso da intervenção, contudo comparando os níveis de incapacidade no momento de admissão e no momento da alta verificam-se melhorias nos últimos sendo 2,8 vezes mais funcionais. 


\section{CONCLUSÕES}

As conclusões deste estudo, vêm reforçar a necessidade de mudança no paradigma da organização dos cuidados de saúde. Estamos de facto perante uma população cada vez mais envelhecida, (53 - 92 anos, $\mathrm{M}=$ 74,83 anos), com graus crescentes de dependência e por essa razão com necessidades de um maior acompanhamento.

A grande maioria $(97,5 \%)$ das pessoas estudadas ao ingressarem na RNCCI apresentava incapacidades funcionais distribuídas entre níveis moderados $(20,0 \%)$ a graves $(77,5 \%)$. Realce-se o facto de no momento da admissão nenhum (0\%) dos elementos apresentar independência funcional total. Contudo e apesar do padrão de dependência se manifestar de forma distinta nos participantes, os ganhos em saúde são efetivos na recuperação da sua funcionalidade, uma vez que, como era espectável, há claras melhorias no nível de dependência e no autocuidado. Veja-se que a independência total foi conseguida por 10,0\% dos utentes e 25\% daqueles que no momento da alta apresentavam incapacidade grave passou a níveis moderados $(30 \%)$ e ligeiros $(7,5 \%)$.

À semelhança de outros estudos constatámos que níveis superiores de escolaridade, rendimentos mensais mais elevados, programas de reabilitação semanais mais continuados $(>10 \mathrm{~h})$ e a manutenção da integridade cutânea, parecem influir de forma positiva e muito significativa ser na capacidade funcional destes utentes, remetendo-nos para a necessidade de uma maior intervenção a estes níveis.

Em síntese, os resultados deste estudo demonstram que o investimento na RNCCI, como um dos pilares do Serviço Nacional de Saúde, numa lógica de continuidade de cuidados, constitui um recurso muito vantajoso para as famílias face ao envelhecimento exponencial da população e consequente aumento do número de pessoas com incapacidade funcional para a realização das suas atividades de vida diárias, permitindo-nos afirmar que o internamento na RNCCI tem impacto positivo na funcionalidade dos Utentes.

\section{Implicações para a prática}

Estas constatações levam-me a reforçar sugestões já apresentadas por outros autores, sobre a importância fulcral e o papel determinante dos profissionais de saúde em especial dos enfermeiros de reabilitação na recuperação de capacidade funcional das pessoas, no contexto do doente internado em UCCI Não temos dúvidas de que os enfermeiros estão numa posição privilegiada de promoção de autonomia e independência 
daqueles que cuidam. Porém e para que isso seja possível, o enfermeiro terá de estar desperto para uma avaliação sistematizada da capacidade funcional pois o conhecimento da sua prevalência é indispensável para determinar recursos e serviços a aplicar, promovendo ambientes facilitadores de autonomia e independência .

\section{REFERÊNCIAS BIBLIOGRÁFICAS}

Barbosa, B. R., (2014). Avaliação da capacidade funcional dos idosos e fatores associados à incapacidade. Ciência \& Saúde Coletiva, 19 (8):3317-3325.

DOI: $10.1590 / 1413-81232014198.06322013$

Decreto-Lei no 265/99. (1999, Julho 14). Criação da Uma nova prestação a pensionistas por Invalidez. Diário da República, 1 (109), pp.38563856. Acedido em

https://www.adse.pt/document/Decreto_Lei_101_2006.pdf

Decreto-lei n. ${ }^{\circ}$ 309-A/2000 de 30 de Novembro (2000). Alterações à lei n. ${ }^{\circ}$ 265/99, de 14 de Julho. Regula a protecção social nas situações que envolvem um grau mais elevado de dependência, complemento por dependência. D.R., $1^{\text {a }}$ Série-A. Acedido em https://dre.tretas.org/dre/123393/

Direção Geral de Saúde. (2013). Plano Nacional de Saúde 2012-2016 Acedido em.

http://1nj5ms2lli5hdggbe3mm7ms5.wpengine.netdnacdn.com/files/20 12/02/Bibliografia-Geral2.pdf

Ferreira, M. S. M.; Pereira, M. G., (2016). O papel moderador do tipo de família na relação entre incapacidade funcional e qualidade de vida em doentes com lombalgia crónica. DOI: 10.1590/141381232015211.01012015

Filipe, M. M. (2015). Contributos para um modelo de contratualização de cuidados em contexto domiciliário: Estudo exploratório sobre os custos de funcionamento das ECCI (Doctoral thesis). Universidade Católica Portuguesa, Instituto de Ciências da Saúde, Porto, Portugal. Recuperado de http://hdl.handle.net/10400.14/20705

Gonçalves, P. J. (2013). Famílias que integram pessoas dependentes no autocuidado: Estudo exploratório de base populacional no concelho do Porto (Doctoral thesis). Universidade Católica Portuguesa, Recuperado de http://hdl.handle.net/10400.14/18580

Instituto Nacional de Estatística (2016). Acedido em 
https://www.ine.pt/xportal/xmain?xpgid=ine_main\&xpid=INE\&xlang $=\mathrm{pt}$

Lino, L. A. V., (2013). Dependência funcional e Auto-estima na pessoa com ferida crónica. Dissertação apresentada à Universidade Católica Portuguesa para obtenção do grau de Mestre em Feridas e Viabilidade Tecidular. Acedido em

http://repositorio.ucp.pt/bitstream/10400.14/11625/1/Disserta\%C3\%A 7\% $3 \%$ A30\% $20-\% 20 \mathrm{MFVT} \% 20-$

$\% 20$ Dependencia\%20Funcional\%20e\%20Auto-

Estima\%20da\%20pessoa\%20com\%20Ferida\%20Cronica.pdf

Lopes, L., (2014) - Necessidades e estratégias na dependência: uma visão da família - VOL. 25, №. 1. Pág. 39-46. Acedido em https://www.ensp.unl.pt/dispositivos-de-apoio/cdi/cdi/sector-depublicacoes/revista/2000-2008/pdfs/03-07.pdf

Lourenço, T. M., (2011). Capacidade funcional do idoso longevo admitido em unidades de internação hospitalar na cidade de Curitiba - PR. Dissertação de Mestrado apresentada ao Programa de PósGraduação Enfermagem, área de concentração, Prática profissional em Enfermagem, Setor de Ciências da Saúde, Universidade Federal do Paraná. Acedido em http://www.ppgenf.ufpr.br/DISSERTACAOTANIALOUREN\%C3\% 87O.pdf

Petronilho, F. A., Pereira, F. M., \& Silva, A. A. (2017). Evolução e destino das pessoas dependentes no autocuidado: Estudo longitudinal. Revista Investigação em Enfermagem, 18, 33-43.

Pinto, E. M., \& Martins, R., (2013). Regresso do doente ao domicílio: Importância do nível de dependência e do apoio familiar. Servir, 58 (1-2), 79-89. Acedido

em http://repositorio.ipv.pt/handle/10400.19/2666

Portugal, Ministério da Saúde (2011). Manual do Prestador Recomendações para a melhoria contínua. Acedido em http://www.acss.min-

saude.pt/wpcontent/uploads/2016/10/Man_Prestador_UMCCIRNCCI.pdf

Portugal, Ministério da Saúde (2016). Administração Central do Sistema de Saúde - ACSS. Monitorização da Rede Nacional de Cuidados Continuados Integrados (RNCCI) $1^{\circ}$ Semestre de 2015. Relatório de monitorização da Rede Nacional de Cuidados Continuados Integrados (RNCCI) $\quad-1^{o}$ Semestre 2015. Acedido: http://www.acss.min-saude.pt/wp 
content/uploads/2016/09/Relatorio_Monitorizacao_RNCCI_1_Semest re-2015.pdf

Saraiva, D. M. F., (2011). O olhar dos e pelos cuidadores: Os impactos de cuidar e a importância do apoio ao cuidador. Tese de Mestrado em Intervenção Social, Inovação e Empreendedorismo, apresentada à Faculdade de Psicologia e Ciências da Educação e à Faculdade de Economia. Vol. I. Coimbra. Acedido em https://estudogeral.sib.uc.pt/bitstream/10316/17858/1/TESE_MISIE_S ET2011.pdf

Sequeira, C. (2007). Cuidar de idosos dependentes. Coimbra: Quarteto Editora.

Veiga, L. M. C.,(2015). Reabilitação funcional após Acidente Vascular Cerebral: resultados de uma Unidade de Convalescença. Apresentado à Escola Superior de Saúde de Bragança - Instituto Politécnico de Bragança, para obtenção do grau de Mestre em Enfermagem de Reabilitação. Acedido em https://bibliotecadigital.ipb.pt/bitstream/10198/11997/1/Liliana\%20M arisa\%20Coimbra\%20Veiga.pdf

Vieira, D.; Freitas, M., Vieira, S. (2015). Programa de Melhoria contínua da qualidade dos cuidados de enfermagem na Unidade de Internamento de Longa Duração Atalaia Living Care - Avaliação do Grau de dependência dos utentes. Caniço. Acedido em http://www.ordemenfermeiros.pt/projectos/Documents/Projetos_Melh oria_Qualidade_Cuidados_Enfermagem/UnidadeInternamentoLonga Duracao_AtalaiaLivingCare_AvaliacaoGrauDependenciaUtentes_Ma deira.pdf

World Health Organization. Euro. (2011). Evaluation of the National Health Plan of Portugal (2004 - 2010). Disponível em http://www.acs.min-saude.pt 